\title{
Design of Self Governing Renewable Energy Residential System by Using Optimization Techniques
}

\author{
G.Vara Lakshmi ${ }^{1}$, V.Usha Reddy ${ }^{2}$ \\ PG student [ICS], Dept. of EEE, S.V.U. College of Engineering, Tirupati, A.P ${ }^{1}$ \\ Assistant Professor, Dept. of EEE, S.V.U. College of Engineering, Tirupati, A.P ${ }^{2}$
}

\begin{abstract}
From past ten years, these self governing residential renewable systems are widely used, especially in rural areas, in isolated areas and archipelagos, the proper design of any renewable energy system and the congeniality of its power quality parameters with IEEE, NEMA and IEC standards are critical for virtuous and thrifty self governing function of the system. To this way, the crucial parameters of a self governing residential renewable systems are appropriately resolved by using Genetic Algorithm and Differential Evolution optimization techniques then design parameters are designed and compared with these two techniques. It is observed from the results that the Differential Evolution algorithm consistently outperforms the GA in terms of both efficiency and the solution quality.
\end{abstract}

Keywords: Differential Evolution, Genetic Algorithm, Photovoltaic Cell, Maximum Power Point Tracking, Inverter.

\section{INTRODUCTION}

The enlargement of electrical energy markets, the increasing rate of electrical power and depreciation of conventional energy resources leads to usage of renewable energy resources especially solar, wind and biogas. Nowa- days as the solar photo voltaic cells are economical and the technological forward in the conversion have forward raised the self governing photo voltaic systems installation. Both economic and impulsive drive consumers to install PV panels and these systems are also financed and supported by the government as the distribution systems are feeble. Power quality is more important for these self governing solar systems as the voltage and current profiles were mainly depends on the radiations and temperature of photo voltaic panels from the sun. As These two parameters were not constant, changes from time to time. For designing of self governing renewable energy systems mainly inverter, battery, capacitor and inductor were used. In this paper, the power quality achieved in standalone PV cells. Renewable energy source is fluctuating dc in nature; the dc-ac inversion followed by ac-dc conversion can be eliminated if the output is dc. Inserting a dc-dc converter with maximum power point tracking (MPPT) capability in between the renewable energy source and the load would save the extra conversion losses associated with the unnecessary conversion stages.

In this paper, we introduce a proper method based on optimization techniques for the design of self governing PV residential systems and the appropriate values for power quality are determined. We mainly used indices are frequency ratio, impedance ratio, transformer turns ratio and DC voltage at the inverter DC side. By using these values we obtain less THD value in the voltage and reducing asymmetry, inverter short-circuit ratio , inverter nominal power ratio, inverter nominal current ratio according to the IEEE, NEMA and IEC standards. An optimization technique in this system is to minimize the objective function which is single objective function. Genetic algorithm and Differential evolution are effective, flexible and powerful, uses guided random search in the population and mutation respectively. Method for solving both constrained and unconstrained optimization problems that is based on natural selection. The algorithm repeatedly modifies a population of individual solutions. Over successive generations, the population "evolves" toward an optimal solution.

\section{PROPOSED SYSTEM}

\section{A. System Configuration}

The proposed system consists of a three phase inverter supplied by a battery bank and through series charger PV unit is charged. We proposed a simple structure for standalone PV residential system. It concentrates both on the transformer and the low pass filter. The dc-ac inversion followed by ac-dc conversion can be eliminated if the output is $\mathrm{dc}$. Inserting a $\mathrm{dc}-\mathrm{dc}$ converter with maximum power point tracking (MPPT) capability in between the renewable energy source can also be used. When it comes to inverter part, we used the standard threephase full bridge inverter because it is apt for residential operation. Any inverter type can be used for proposed optimum technologies. Moreover, the transformer turns ratio affects the selection of the DC voltage level because at the load side depends on turns ratio.

The formulae which are used:

$$
\begin{gathered}
V_{i n, N}=\sqrt{3 / 2} m a \frac{V_{d c}}{2} \\
\bar{V}_{1 N}=\bar{V}_{i n v, n}-\Delta \bar{V}_{L f} \\
V_{2 N}=\frac{\sqrt{3} V_{1 N}}{n}
\end{gathered}
$$

Where $(1,2,3)$ denotes inverter nominal rms current 
transformer line to- line nominal primary voltage, transformer line to -line nominal secondary voltage respectively. These are derived from SPWM inverter .The bar indicates phasor quantities. The circulation of zero sequence components is blocked at the load side as the transformer is Ygd connection and triple order harmonic current components are also blocked because of non linear loads. Such that Here for optimization we used the objective function as the

$$
e(k)=\frac{a s y m}{\omega_{1}}+\frac{t_{5}}{\omega_{2}}+\frac{t_{7}}{\omega_{3}}+\frac{t_{11}}{\omega_{4}}+\frac{s c i}{\omega_{5}}+\frac{s c s}{\omega_{6}}+\frac{n o m i}{\omega_{7}}
$$

According to IEEE, NEMA and IEC standards these values are defined and limits for these parameters are tabular columned.

TABLE I: Parameter's Limitations

\begin{tabular}{|c|c|}
\hline parameter & Limit (\%) \\
\hline Asym & $<5$ \\
\hline THDv & $<8$ \\
\hline Scs & $<2$ \\
\hline nomi & $<1.5$ \\
\hline Sci & $<5$ \\
\hline
\end{tabular}

The limits for asym and $T H D_{v}$ parameters have been obtained by NEMA and IEC Standards. Additionally, the selection for sci, scs, and nomi limits in Table I has been based on techno-economical criteria (commercial inverters safe operating area). Due to the large number, complexity, and nonlinearity of the derived equations, an optimization techniques a genetic algorithm (GA) and differential evolution are applied for the determination of the parameters values. In this paper, an appropriate GA is selected, which has produced excellent results in similar optimization problems, in order to adjust the parameters $\mathrm{f}$, $\mathrm{q}, \mathrm{n}$ and $V_{d c}$, so as to minimize the optimization functione $(k)$.

Where $w_{1,2,3 \ldots n}$ are weight factors, set to the upper limits of Table I for normalizing all the design parameters. $t_{5}, t_{7}, t_{11}$ and are computed considering nonlinear loads with harmonic current content equal to the maximum permissible limits for each harmonic component, according to IEC 61000-3-2 standard. They are defined as

$$
t_{i}=\frac{X \cdot Z_{i} \cdot I_{i}}{230}
$$

Where $\mathrm{i}=5,7,11$ harmonic order

Thus, according to Table I, the weight factors take the following values $\mathrm{w} 1=0.05, \mathrm{w} 2=0.06, \mathrm{w} 3=0.05$, w $4=0.035$, w5 $=5$, w6 $=2$ and $\mathrm{w} 7=1.5$

$$
k=\left[k_{1}, K_{2}, K_{3}, K_{4}\right]^{T}=\left[f, q, n, V_{d c}\right]^{T}
$$

Where $\mathrm{k}$ is a column vector containing variables $\mathrm{K} 1$, $\mathrm{K} 2, \mathrm{~K} 3, \mathrm{~K} 4$

The terms in the objective function are follows

a) Asymmetry due to Single-Phase Loads (asym): true definition of the voltage unbalance is as follows

$$
\begin{aligned}
& \text { asym }=\frac{V_{2}}{V_{1}} \cdot 100 \% \\
& =\left\langle\left\{1+\left[\left(\frac{f_{r}}{f_{b}}\right)^{2}-1\right] \frac{n^{2} R_{L}}{X_{C f}} \tan [\operatorname{arcos}(p f)]\right\}^{2}+\left\{\left[\left(\frac{f_{r}}{f_{b}}\right)^{2}-1\right] \frac{n^{2} R_{L}}{X_{C f}}\right\}^{2}\right\rangle^{-\frac{1}{2}} .100 \%
\end{aligned}
$$

Where $f_{r}=$ filter resonant frequency, $f_{b}=$ load voltage angular frequency, $R_{L}=$ load impedance resistive part, $\mathrm{n}=$ transformer turns ratio, $X_{C f}=$ filter capacitance impedance, $V_{1}=$ positive sequence voltage component , $V_{2}=$ negative sequence voltage component.

Where asym should vary within the limits imposed by the IEC, NEMA, and IEEE(national and international standards) even for the worst load case scenario.

b) Total Harmonic Distortion Due to Nonlinear Loads $\left(T H D_{v}\right)$ : The load voltage harmonic distortion due to the existence of nonlinear loads especially three phase non linear loads is considered, due to their higher expected impact on load voltage waveform. The value has to be also within the limits levied by the NEMA,IEEE and EN 50160, for the worst load case scenario also.

c) Inverter Short-Circuit Current Ratio (sci): This factor is defined as

$$
S C i=\frac{I_{i n, s c}}{I_{i n, N}}
$$

sci denotes the short-circuit current that both the inverter and the filter inductor have to withstand.

d) Inverter Nominal Power Ratio (scs): This factor is defined as

$$
\begin{aligned}
S C S & =\frac{S_{i n, N}}{S_{L, N}} \\
S_{i n, N} & =\sqrt{3} V_{i n, N} I_{i n v, N} \\
S_{L, N} & =\sqrt{3} V_{2 N} I_{2 N}=\frac{P_{L}}{P_{f}}
\end{aligned}
$$

Where scs is a techno-economical factor defines the essential inverter over dimension in order to meet the power quality targets and the inverter safe operation restrictions.

e)Inverter Nominal Current Ratio (nomi): This factor is defined as

$$
n o m i=\frac{I_{i n v, N}}{I_{1 N}}
$$

nomi denotes the maximum permissible capacitive current in the circuit, being a restriction for the determination of and values.

\section{III.OPTIMIZATION TECHNIQUES}

\section{A) Genetic Algorithm:}

Genetic Algorithm (GA) is a global optimization algorithm derived from evolution and natural selection.it is a search technique used in computing to find true/approximate solutions to optimization and search problems. Though genetic algorithm cannot always provide optimal solution, it has its own advantages and is a powerful tool for solving complex problems.Genetic algorithms are modelled by the genetic information of each individual or potential solution is encoded in structures called chromosomes. In genetic algorithm, coding is expressing the individual by the binary strings of $0 \mathrm{~s}$ and $1 \mathrm{~s}$. In the instance one, every individual has three 
dimensions, and every dimension is expressed by a 8- bit string of $0 \mathrm{~s}$ and $1 \mathrm{~s}$, so every individual is expressed as a 24-bit string of 0 s and $1 \mathrm{~s}$, showing as

\section{0}

Here individual corresponds to any possible solution that defines population as group of all individuals. The genetic operator used to vary the generation of a chromosome from one generation to another is defined as cross over. After selection and crossover have a new population full of individuals some are directly copied and some are produced by crossover. In order to ensure that the individuals are not all exactly the same it allows for a small chance of mutation.

\section{The basic steps of Genetic algorithm:}

1) Randomly producing a original population whose number of individuals is a constant $\mathrm{N}$.

2) Producing next generation by crossing over and mutation among individuals.

3 ) Forming the new population of $\mathrm{N}$ individuals from the generation of 2)

4) Producing the next population by repeating the step2) and 3) until obtaining the individual which satisfies conditions.

\section{B) Differential Evolution}

Objective functions that are non differentiable, noncontinuous, non-linear, noisy, flat, multi-dimensional or have many local minima, constraints or stochasticity. Such problems are difficult if not impossible to solve analytically those can be easily solved by Differential evolution.DE is an Evolutionary Algorithm. This class also includes Genetic Algorithms, Evolutionary Strategies and Evolutionary Programming. DE starts with a fixed population size of NP candidate solutions which may be represented as $\mathrm{Xi}, \mathrm{G}, i=1, \ldots, \mathrm{NP}$, where $i$ index denotes the population and $G$ denotes the generation to which the population belongs. The working of DE depends on the efficiency of three main operators; mutation, reproduction and selection which briefly described in this section.

Mutation: Mutation operator is the prime operator of DE and it is the implementation of this operation that makes DE different from other Evolutionary algorithms. The mutation operation of DE applies the vector differentials between the existing population members for determining both the degree and direction of perturbation applied to the individual subject of the mutation ope ration. The mutation process at each generation begins by randomly selecting three individuals in the population.

Crossover: once the mutation phase is complete, the crossover process is activated. The perturbed individual, $\mathrm{Vi}, \mathrm{G}+1=(\mathrm{v} 1, \mathrm{i}, \mathrm{G}+1, \ldots, \mathrm{vn}, \mathrm{i}, \mathrm{G}+1)$, and the current population member, $\mathrm{Xi}, \mathrm{G}=(\mathrm{x} 1, \mathrm{i}, \mathrm{G}, \ldots$. $\ldots \mathrm{xn}, \mathrm{i}, \mathrm{G})$, are subject to the crossover operation, that finally generates the population of candidates, or "trial "vectors

$$
\mathrm{Ui}, \mathrm{G}+1=(\mathrm{u} 1, \mathrm{i}, \mathrm{G}+1, \ldots, \mathrm{un}, \mathrm{i}, \mathrm{G}+1)
$$

Thus, each individual of the temporary (trial) population is compared with its counterpart in the current population. The one with the lower objective function value will survive from the tournament selection to the population of the next generation. As a result, all the individuals of the next generation are as good or better than their counterparts in the current generation. In DE trial vector is not compared against all the individuals in the current generation, but only against one individual, its counterpart, in the current generation.

The algorithm steps are given here

\section{1) DE Algorithm steps}

Step 1: The random initialization of the parent population. Randomly generate a population of (say) NP vectors, each of $\mathrm{n}$ dimensions:

$$
x i, j=x \min , j+\operatorname{rand}(0,1)(x \max , j-x \min , j),
$$

Where $x \min , \mathrm{j}$ and $\mathrm{xmax}$ are lower and upper bounds for jth component respectively, $\operatorname{rand}(0,1)$ is a uniform random number between 0 and 1 .

Step 2: Calculate the objective function value $\mathrm{f}(\mathrm{Xi})$ for all $\mathrm{Xi}$.

Step 3: Select three points from population and generate perturbed individual $\mathrm{Vi}$

Step 4: Recombine the each target vector xi with perturbed individual generated in step 3 to generate a trial vector Ui using equation (2).

Step 5: Check whether each variable of the trial vector is within range. If yes,

then go to step 6 else make it within range using

ui, $\mathrm{j}=2 * x \min , \mathrm{j}-\mathrm{ui,j}$,

if ui,j<xmin,j and ui,j $=2 * x \max , \mathrm{j}-\mathrm{ui,j}$, if ui,j> $\mathrm{xmax}, \mathrm{j}$, and go to step 6.

Step 6: Calculate the objective function value for vector Ui.

Step 7: Choose better of the two (function value at target and trial point) for next generation.

Step 8: Check whether convergence criterion is met if yes then stop; otherwise go to step 3

\section{IV.OPTIMIZATION RESULTS}

\section{A) Genetic algorithm results}

Two self governing system power levels are studied as typical examples of residential applications, i.e., 20 and 50 $\mathrm{kW}$ representing the maximum load of a single residence and a small hotel, respectively. In all cases, the load power factor is set 0.85 , whereas the load voltage asymmetry (i.e., single-phase loading rated at its nominal value per phase) is calculated for the worst case scenario, i.e., single-phase loading rated at its nominal value (per phase).

The optimal values for the design parameters are shown in Figs. 1-4 as functions of the -value.

It is noted that the $\mathrm{X}$-value increase leads to an analog increase of the harmonic components of the load current. On the other hand, the load active power remains constant to its nominal value (20 or $50 \mathrm{~kW})$.

Another point that has to be clarified is the selection of, $\mathrm{m}_{\mathrm{a}}$ -value under steady-state operation. As reported in Figs. $1-4$, in all cases, is set to 0.5 ; this is a typical value based on the SPWM inverter linear operation area. 


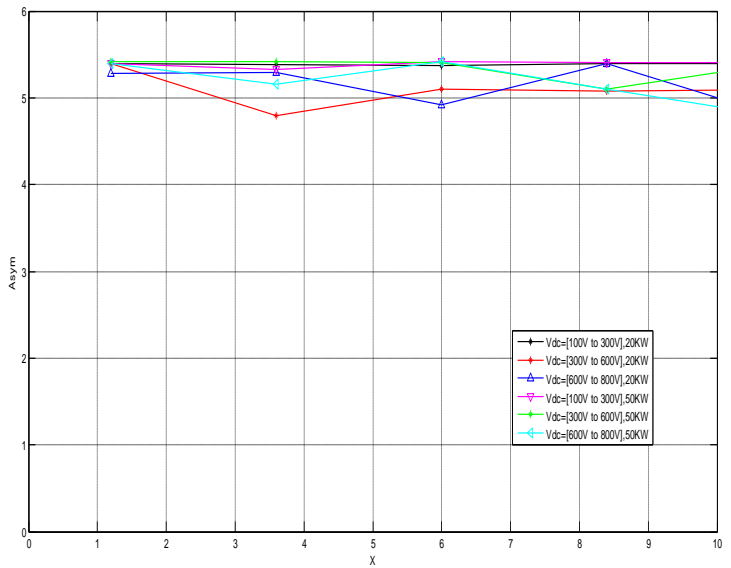

Fig:1:asym vs x non linear loads

Obviously, the optimization can be performed under any desirable $\mathrm{m}_{\mathrm{a}}$-value.By studying the outcomes of the optimum design, it can be deduced that it is feasible to set the values of the key parameters with respect to the limitations of Table I, ensuring the safe operation of an self governing PV system, whereas avoiding overdimensioned designs and non practical theoretical solutions. Especially for the parameter, the results show that the proposed design limits its value at significantly lower levels than the one imposed by the EN 50160 standard. So, the necessary effort in terms of voltage controller design is significantly reduced.



Fig.2:THD vs $\mathrm{X}$ non linear loads

As it concerns the maximum expected load voltage asymmetry, it can be observed that it beyond's in some cases the limit of 5\%. This is due to the fact that the GA has been applied without imposing any restrictions on the extracted values of the design parameters. So, by the optimization results, the asym parameter slightly varies between $4.8 \%$ and $5.4 \%$, and it does not actually depend on $\mathrm{X}$-value.

This uncommon behavior is again examined by repeating the optimization process and imposing a 5\%limit for the asym parameter; the main effect of this restriction is the increase of the scs value,Since the load voltage asymmetry stands for an extremely seldom situation (i.e., only one phase is loaded at its rated power), it is deduced that the results in Fig. 1 are not on the safe side-the load voltage asymmetry higher than $5 \%$ limit.

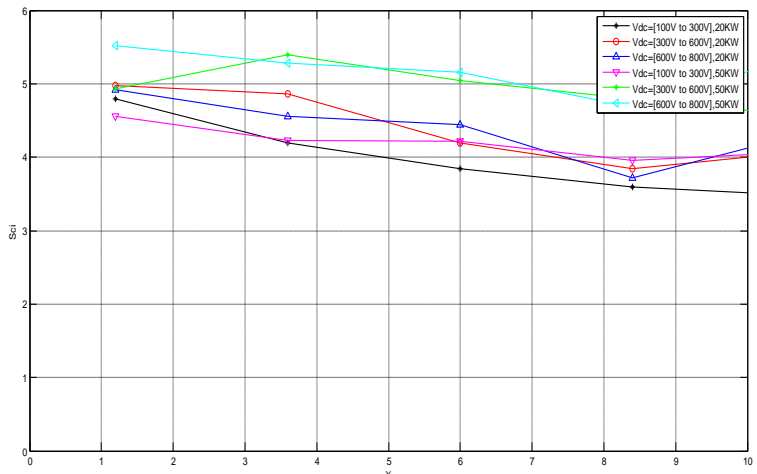

Fig .3:SCI Vs X non linear loads

Furthermore, sci and scs parameters present similar behavior as well and importantly, in some cases, the results overrun slightly the defined restrictions without affecting the power quality of the PV system or leading to non realistic designs. On the other hand, nomi overruns the prescribed limit in Table I, for $\mathrm{X}>4$ - and 20-kW load case. High nomi values lead to increased capacitive currents (higher capacitances) in the low-pass filter. Thus, the inverter installed power becomes higher due to the excessive capacitive reactive power. the results in Fig. 3 show's clearly that the proposed optimization strategy manages to keep the inverter over-dimensioning not within the acceptable range (as indicated by sci and scs limits). Once again, it has to be noted that this inconvenient situation stands only for extremely distorted load and for lower power installation. However, in more realistic conditions $(X<4)$, nomi remains within the prescribed limit.

Another concept that has to be discussed is the effect of the load nominal power value. The optimization results in Figs. 1-4 highlight the fact that $T H D_{v}$, scs, and nomi have lower values in $50-\mathrm{kW}$ case. Actually, the values of these parameters are below the prescribed limits even for $X=9$. This outcome makes sense by taking into account that the harmonic components of the load current are at the same absolute levels for both load conditions

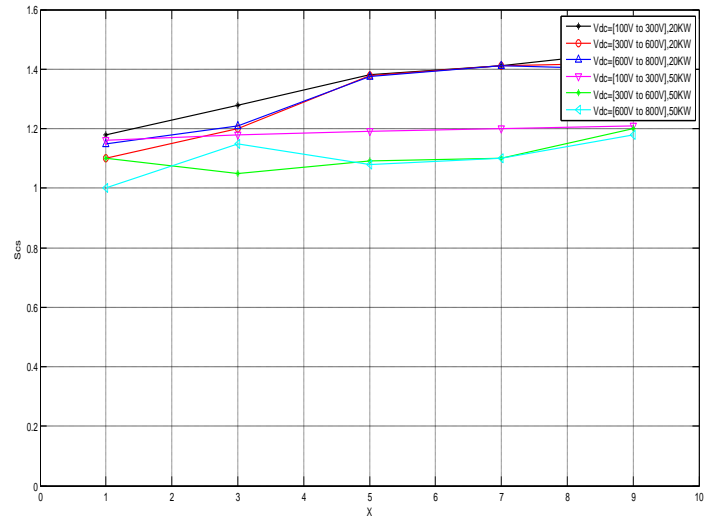

Fig.4:SCS vs X non linaer loads

Thus, the effect of the harmonic current content to the load voltage quality is reduced in the $50-\mathrm{kW}$ case-leading so to reduce $T H D_{v}$-value. Significantly, scs and nomi parameters become also reduced because lower inverter installed power is now necessary to meet the power quality targets. On the contrary, both asym and sci parameters do 
not present any particular relation with the load level, because both the numerators and the denominators are proportional to the load power. Therefore, the derived results show clearly the effectiveness of the proposed methodology in terms of high power quality.



Fig.5:Nomi vs X non linear loads

Then for differential evolution results are as follows.

\section{b) Differential evolution results}

The results here shown are more and farther best as the values shows the limited values as per international and national standards

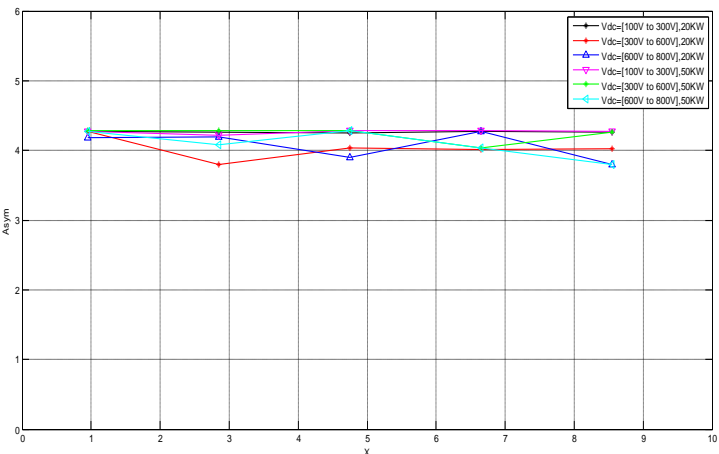

Fig.6: asym vs X non linear loads for DE

as in the figure 6 shows the asymmetry values are below the limits of $<5$. This shows the number of iterations are high and by searching vast area we get excellent results for DE.

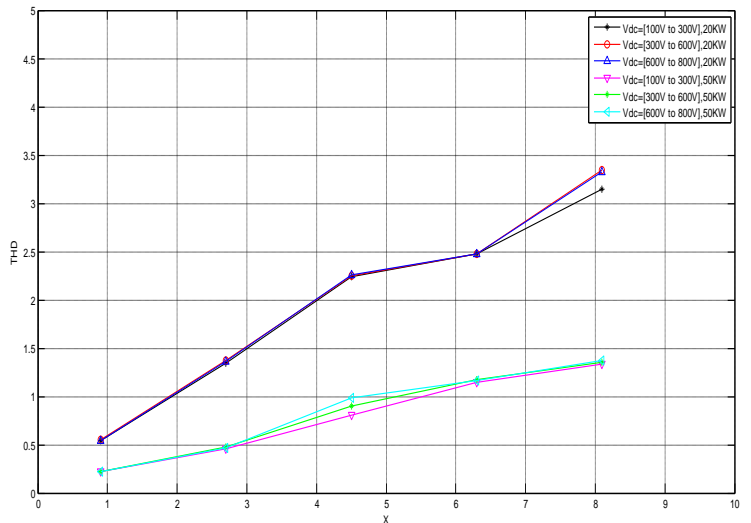

Fig.7:THD vs X non linear loads

Thus, the effect of the harmonic current content to the load voltage quality is reduced in the $50-\mathrm{kW}$ case-leading so to reduce $T H D_{v}$-value.

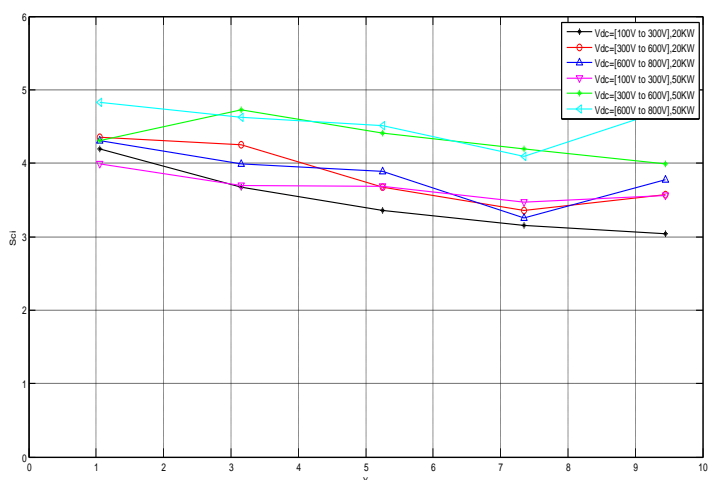

Fig.8:SCI vs X non linear loads

Moreover, sci and scs parameters present improved behavior as well and importantly,these values are in the limits tabular column I .

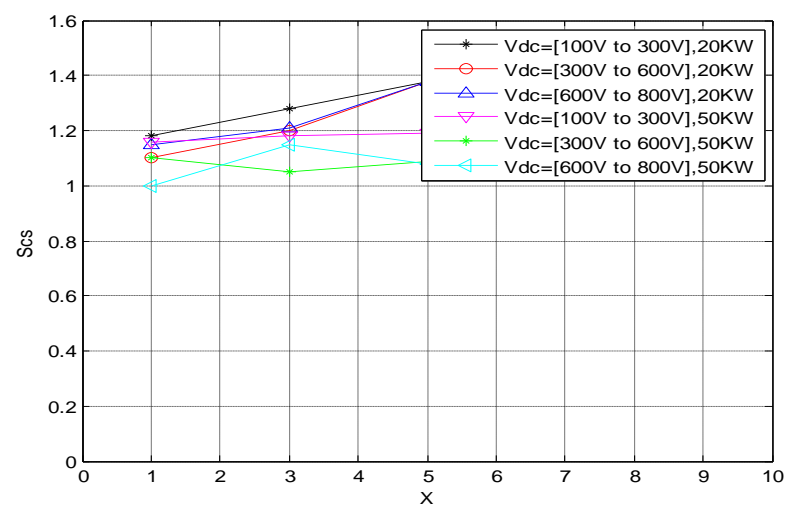

Fig.9:SCS vs X non linear loads

As it concerns the application of the proposed design method, the maximum load of the standalone installation and the major nonlinear loads (e.g., inverter drives, high power rectifiers) have to be determined. The $p_{f}$ value may be set to a worst case value (between 0.75 and 0.85 ) if it is difficult and/or impossible to make precise calculations. According to the above data, the values of $S_{L, N}, X$, parameters are evaluated for the maximum load active power. It is noted that the calculation of $\mathrm{X}$-value corresponds to the total harmonic content that the major nonlinear components inject. Next, the optimization algorithm can be directly applied and hence the values of $\mathrm{n}$, $\mathrm{q}$, and $\mathrm{f}$ parameters can be extracted. This is a repeatable process with $V_{d c}$ and ma being.

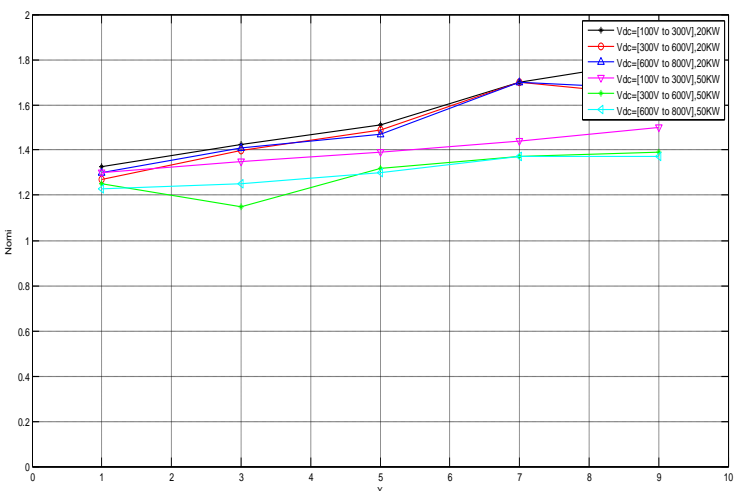

Fig.10:Nomi vs X non linear loads 
As both results shows that the DE technique has better convergence properties than all the GAs. The DE technique could locate the current best solution with a higher frequency than the GAs in all cases. DE is also robust; it is able to reproduce the same results over many trials, whereas the GA performance is more dependent on the randomized initialization of the individual parameters. A genetic algorithm method uses a strategy that generates variations of the design parameter vectors. Once a variation is generated, the new parameter vector is accepted or not. The new parameter vector is accepted in the case it reduces the objective function value. The genetic algorithm converges fast but can be trapped by local minima. This disadvantage can be eliminated by running several vectors simultaneously. This is the main idea of Differential Evolution (DE) algorithm.

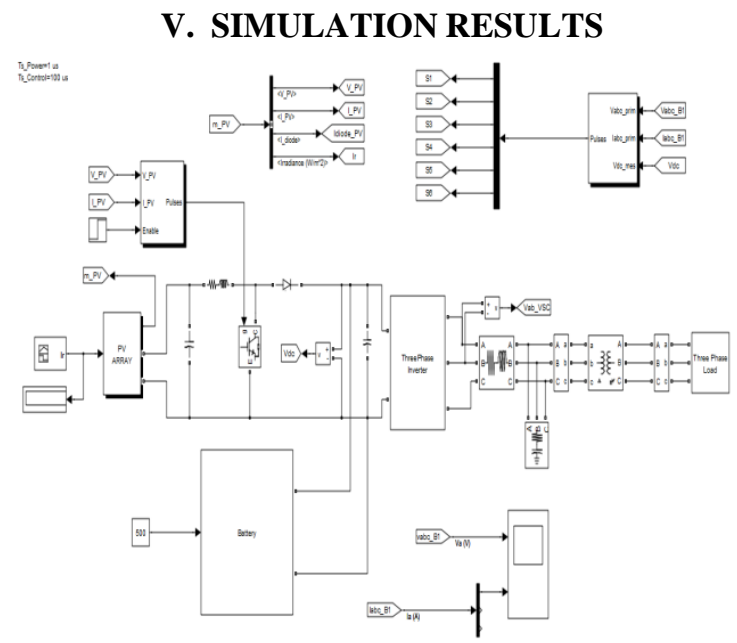

Fig.11: Simulation of circuit PV system for three phase load using non linear load.

The proposed system method is simulated in MATLAB for the voltage and current waveforms using non linear load .The results are showing for THD (v). The second order harmonic current waveforms are obtained for three phase PV system. For single phase loads ground connector is used as the neutral line. We used $50 \mathrm{KW}$ i.e., $\mathrm{Vdc}=500 \mathrm{~V}$ and for $20 \mathrm{KW}$ used single phase load.
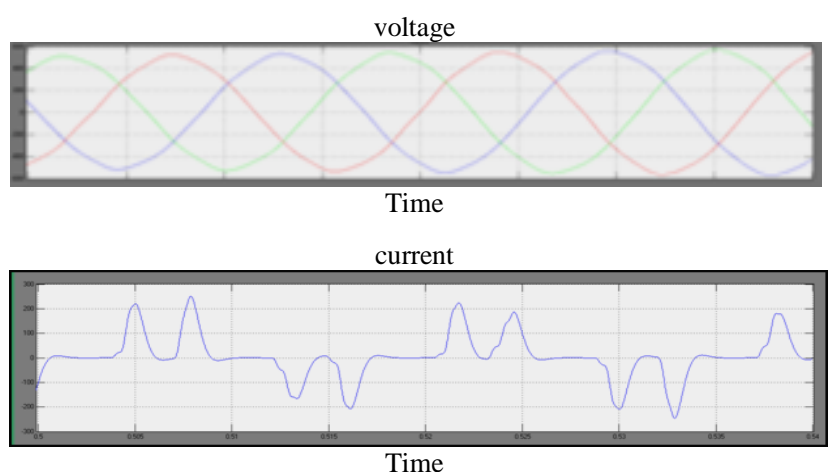

Fig.12:Load voltage waveform and current waveform for 50 $\mathrm{KW}$ three-phase diode rectifier using $3 \mathrm{mF}$ smoothening capacitor

Simulink block model of the system under study and the Gnd-connector is used as the neutral line for the connection of single-phase loads. Of course, the range of realistic values for each variable is set by the designer as well as by technical restrictions. For example, ma has to be lower than one (otherwise over modulation issues rise) and $\mathrm{V}_{\mathrm{dc}}$ is usually between 100 and $800 \mathrm{~V}$ in commercial inverters for standalone installations. The final selection of $\mathrm{Vdc}, \mathrm{ma}, \mathrm{n}, \mathrm{q}$, and $\mathrm{f}$ values will be one that meets all the power quality targets given in Table I. Thus, all the technical characteristics of the self governing installation can be calculated (Lf, Cf, $\mathrm{S}_{\mathrm{inv}, \mathrm{N}}, \mathrm{V}_{1 \mathrm{~N}}$ and) by using their definitions. So, the system designer may proceed with the selection of the suitable commercial inverter, transformer unit, and battery bank configuration. Next, an example for the application of the proposed design will be presented.



Fig.13: Showing THD value for voltage waveform

\section{CONCLUSION}

Optimization methods Genetic Algorithm and Differential Evolution for the design of self governing residential PV systems with improved power quality (under steady-state conditions) have been introduced in the present paper. The results has indicated that the power quality behaviour of a standalone residential PV system is strongly dependent on the adjustment of significant factors that must be appropriately selected in order to meet the standards with the IEEE, NEMA and IEC standards. Comparing both techniques Differential Evolution algorithm consistently outperforms the Genetic Algorithm in terms of both efficiency, time and the solution quality.

\section{REFERENCES}

1) Miller, T. J. E., editor,Reactive Power Control in Electrical Systems New York: JohnWiley \& Sons, 1982.

2) NEMA Standards Publication No. 250-1 985, Enclosures for Electrical Equipment (1 000 VoltsMaxi mum)

3) S. Rahman, "Green power: What is it and where can we find it?" IEEEPower Energy Mag., vol. 1, no. 1, pp. 30-37, Jan./Feb. 2003

4) J. A. Gow and C. D. Manning, "Photovoltaic converter system suitable for use in small scale stand-alone or grid connected applications," Proc.Inst. Electr. Eng.-Electr. Power Appl., vol. 147, no. 6, pp. 535-543,Nov. 2000

5) F. Valenciaga and P. Puleston, "Supervisor control for a standalone hybrid generation system using wind and photovoltaic energy," IEEE Trans. Energy Convers., vol. 20, no. 2, pp. 398-405, Jun. 2005.

6) C. Wang and M. Nehrir, "Load transient mitigation for stand-alone fuel cell power generation systems," IEEE Trans. Energy Convers., vol. 22, no. 4, pp. 864-872, Dec. 2007.

7) R. Wai, W. Wang, and C. Lin, "High performance stand-alone photovoltaic generation system," IEEE Trans. Ind. Electron., vol. 55 , no. 1 , pp. 240-250, Jan. 2008

8) 8 J. Bialasiewicz, "Renewable energy systems with photovoltaic power generators: Operation and modeling," IEEE Trans. Ind. Electron., vol. 55, no. 7, pp. 2752-2758, Jul. 2008.

9) 5. N. Stretch and M. Kazerani, "A stand-alone, split-phase currentsourced inverter with novel energy storage," IEEE Trans. Power Electron., vol. 23, no. 6, pp. 2766-2775, Nov. 2008. 
10) L. Wang and D. Lee, "Load-tracking performance of an autonomous SOFCbased hybrid power generation/energy storage system," IEEE Trans. Energy Convers., vol. 25, no. 1, pp. 128-139, Mar. 2010.

11) H. Fakha, D. Lu, and B. Francois, "Power control design of a battery charger in a hybrid-active PV generator for load-following applications," IEEE Trans. Ind. Electron., vol. 58, no. 1, pp. 85-94, Jan. 2011.

12) X. Li, D. Hui, and X. Lai, "Battery energy storage station (BESS)based smoothing control of photovoltaic (PV) and wind power generation fluctuations," IEEE Trans. Sustain. Energy, vol. 4, no. 2, pp. 464-473, Apr. 2013

13) S. Kim, J. Jeon, C. Cho, J. Ahn, and S. Kwon, "Dynamic modeling and control of a grid-connected hybrid generation system with versatile power transfer," IEEE Trans. Ind. Electron., vol. 55, no. 4, pp. 1677-1688, Apr. 2008.

14) H. Geng, D. Xu, B. Wu, and W. Huang, "Direct voltage control for a standalone wind-driven self-excited induction generator with improved power quality," IEEE Trans. Power Electron., vol. 26, no. 8, pp. 2358-2368, Aug. 2011.

15) M. Hamzeh, A. Ghazanfari, H. Mokhtari, and H. Karimi, "Integrating hybrid power source into an islanded MV micro grid using CHB multilevel inverter under unbalanced and nonlinear load conditions," IEEE Trans. Energy Convers., vol. 28, no. 3, pp. 6

16) O.Amanifar and M.E.Hamedani Golshan, "Optimal Distributed Generation Placement and Sizing for Loss and THD Reduction and Voltage Profile Improvement in Distribution Systems using Particle Swarm Optimization and Sensitivity Analysis", International Journal on Technical and Physical Problems of Engineering, Vol. 3, No. 2, pp. 47,48,51,53, 2011

17) Liu yong, Kang lishan \& Chen yuping. (1997). Nonnumerical parallel algorithm(second volumn)_-genetic algorithm. Beijing:science press, 1997 